DOI: https://doi.org/10.14311/TPFM.2021.007

\title{
HOW MANUFACTURING INACCURACIES AFFECT VORTICES IN AN AIRFOIL WAKE
}

\author{
Daniel Duda 1 \\ ${ }^{1}$ Faculty of Mechanical Engineering, University of West Bohemia in Pilsen, Univerzitní 22, \\ 30614 Pilsen, Czech Republic
}

\begin{abstract}
We investigate the statistics of individual vortices found in the wake past airfoil NACA 64-618 (or 643618). We use two realizations of this airfoil: the first one is created by a 3D print of the profile downloaded from public database. By using a 3D scanning technology (GOM Atos), we detect the differences from the design geometry. We prepare a better product, which is closer to the design geometry, but still, it is rather far from ideal case. The flow wake past these two airfoils is measured by using Particle Image Velocimetry (PIV). We find the individual vortices in the instantaneous $2 \mathrm{D}$ velocity fields. We compare the statistical distributions of the vortex core radii and distance to nearest other vortex. Probability density function (PDF) of core radius scales as $R^{1.6}$ and $R^{-2}$, while the PDF of distance to nearest vortex does as $D^{3 / 4}$ and $D^{-4}$. We compare two different realizations of NACA 64-618 airfoil at Reynolds number $3.1 \cdot 10^{4}$ and zero angle of attack.
\end{abstract}

Keywords: Particle Image Velocimetry, Turbulence, Vortex, Wake, NACA 64-618, 3D scanning, GOM Atos.

\section{Introduction}

Vortex is a naturally three-dimensional structure, which has shape of infinite path worming through the fluid or creating closed loops or ending at the boundaries of the fluid. This is valid only in the case of superfluid turbulence [1,2]. In the classical fluids [3], the vorticity is distributed within a vortex tube, their circulation not need to be constant along its path due to the viscous damping effects [4]. And even the vortex core itself can become turbulent containing other vortices [5].

The velocity in turbulent flow is determined by the induction by all present vortices, or vortex tubes, while these vortices are carried by the velocity of the fluid they exist in. This quite simple scheme leads to the idea of Vortex Filament Method [6], which is a numerical integration scheme for computational solving of superfluid flows in the limit of absolute zero, although the finite temperatures can be taken into account [7]. In classical fluids, this method has much more quests to solve $[8,9]$, which is little bit easier in 2D flows [10].

We are experimentalists and we try to explore the fascinating nature of turbulent flows oppositely - we try to find the individual vortices within the experimentally measured turbulent velocity fields. This task has been solved many times in the history, but in most cases, the authors try to search the vortices as a local maxima in some scalar field [11]. E.g. Ben-Gida [12]detects maxima of $\lambda_{c i}$ in a wake past accelerating hydrofoil in stratified or non-stratified water. Kolár [13] developed probably the best scalar function for detecting vortex, Maciel [14] improved it by using eigen decomposition instead of Koláŕ's numerical search of best angle for triple decomposition among rigid-body rotation, pure shear and irrotational strain. However, there are few attempts to solve this problem topologically $[15,16]$, as there is not yet some satisfactory definition of vortex [17].

Our individual vortex searching algorithm is based on direct fitting of the $2 \mathrm{D}$ instantaneous velocity field obtained by Particle Image Velocimetry (PIV) measuring method. Therefore, the vortices are represented as located point-object (in 3D space, they are a linear objects without fully localization) represented by four parameters: position in plane ( $x$ and $y$ ), vortex core radius $R$ and circulation $\Gamma$. The fitting procedure looks for local minimum of residual energy of instantaneous velocity field after subtracting the velocity field induced by the vortex of fitted parameters. More details about the fitting algorithm and starting positions are in our previous article [18]. We already used this algorithm for the flow inside a single-stage axial turbine [19]. The three-dimensional 
effects are not taken into account at this stage of research, however, it will be a great task to expand this analysis method into the $3 \mathrm{D}$ velocity data obtained e.g. by Volumetric PIV ${ }^{1}$.

\section{Experimental setup}

\subsection{Flow measurement by PIV}

The Particle Image Velocimetry (PIV) method measures the movement of small particles carried by the flow $[20,21]$. The tracer particles are produced by a commercially available fog generator Safex, whose usage for air has been proven by experience $[22,23,24]$. The particles are illuminated by a solid-state laser New Wave Solo and captured by a double-frame camera FlowSense MkII of resolution $2048 \times 2048$ pixels. The rough images are processed by using commercial software Dantec Dynamic Studio, where we apply the function Adaptive PIV getting instantaneous velocity fields of resolution $64 \times 64$ grid points.

The field of view is located just behind the airfoil trailing edge and it has dimension $34 \times 34 \mathrm{~mm}=$ $0.42 \times 0.42 c$, where $c$ is the chord length; however, we use only $62 \times 62$ grid points, as the vectors at the edge of the field of view are not trustworthy, because there is lot of particles leaving or entering the field of view between the two laser pulses. The field of view is oriented along the main flow and perpendicularly to the height of the airfoil. Its distance from the wind tunnel walls is $80 \mathrm{~mm}=1 \mathrm{c}$ and $45 \mathrm{~mm}=0.6 c$ respectively.

\subsection{Airfoil creation and control of the outcome}

The NACA 64(3)-618 belongs to the 6-digit NACA group; it is highly non-symmetrical profile, which has reasonable flow characteristics even at high angles of attack, but this is valid at large enough Reynolds numbers. This profile is used at the tip of wind turbine blades. Du et al. [25] used computational fluid dynamics (CFD) to investigate the performance of several airfoil profiles including NACA $64-618$ at angles of attack up to $40^{\circ}$ at Reynolds number $1.15 \cdot 10^{7}$ and $3.3 \cdot 10^{4}$.

We downloaded the profile data from an open-access database Airfoil Tools [26], where it is indexed as NACA 64(3)-618 (naca643618-il) ${ }^{2}$. We create this airfoil by using the Prusa Mk 2.5 3D printer from polymerized lactic acid (PLA) [27].

(a) Top view

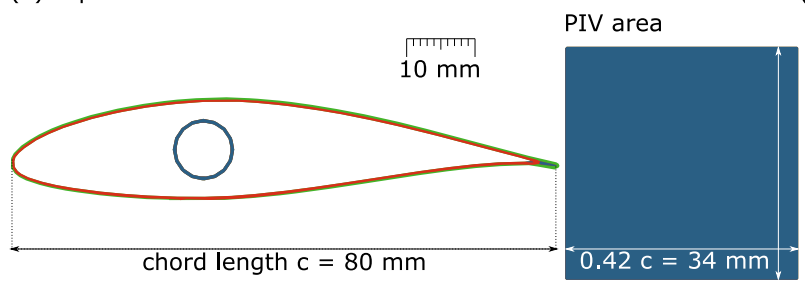

(b) Detail of trailing edge of $A$ with sections of $B$ and $C A D$

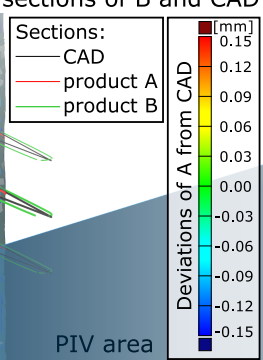

Figure 1: (a) the sections of airfoil with the area studied by using PIV; the sections are distinguished by color: black represents ideal geometry, red does the scan of product A and green does that of product $\mathrm{B}$. (b) the $3 \mathrm{D}$ view on the scan of trailing edge of the product $\mathrm{A}$ colored by the local deviations from the ideal geometry. We plot there the sections in the height of PIV plane and $2 \mathrm{~mm}$ above and under it. CAD abbreviates Computer Aided Design.

The deviations caused by the finite precision of 3D printing technology have been investigated by using a commercial optical [28] 3D scanner GOM ATOs CoRE with measuring volume $300 \mathrm{~cm}^{3}$. Figure 1 shows the scan, panel (a) shows the sections of both discussed products as well as the ideal geometry, while the panel (b) zooms to the trailing edge of the product A colored by the distances from the ideal geometry. There are several sections displayed as well. The main discrepancy in

\footnotetext{
${ }^{1}$ which is in fact PTV - Particle Tracking Velocimetry

${ }^{2}$ http://www.airfoiltools.com/airfoil/details?airfoil=naca643618-il
} 
the product $\mathrm{A}$ is the missing trailing edge, the airfoil is shorter by almost $2 \mathrm{~mm}$ ! The reason for this huge mistake was in the g-code ${ }^{3}$ generation procedure, which avoided plastic extrusion in the area of too thin trailing edge. Thus the geometry for product B has been expanded by using the Minkowski sum [29] with circle of radius $0.1 \mathrm{~mm}$. Then the extruder moved into the thin trailing edge as well. On the other hand, the product was slightly larger than it should be (by the circle of $0.1 \mathrm{~mm}$ radius). Better result has been obtained later at product denoted $\mathrm{C}$, which is not discussed in this article. However, the production of so thin trailing edge with the 3D printing technology is very difficult and another fabrication technology might be taken into account.

\section{Results}

\subsection{Average quantities of velocity fields}
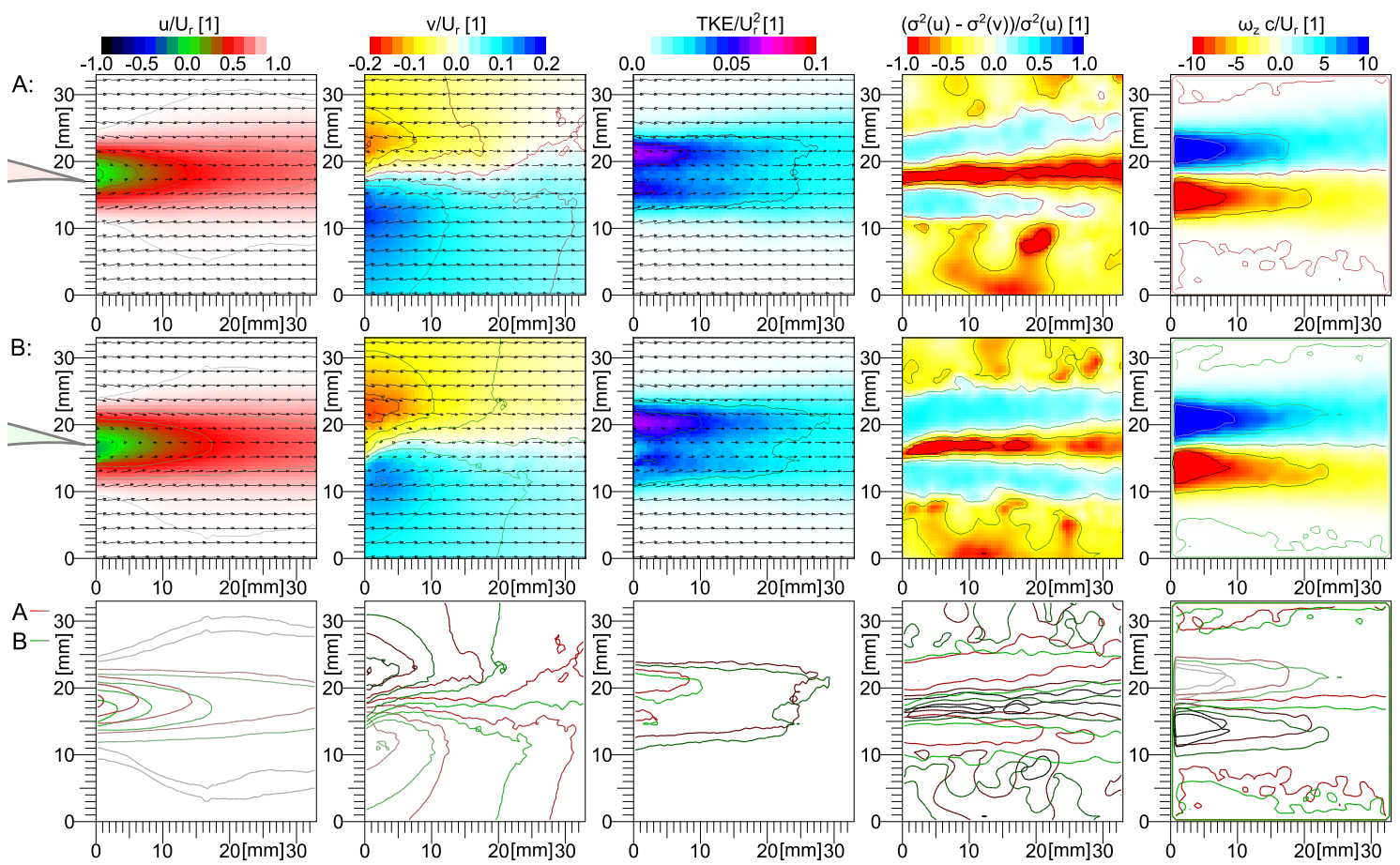

Figure 2: Spatial distribution of ensemble averages of stream-wise velocity (first column), span-wise velocity (second column), in-plane turbulent kinetic energy (third column), degree of anisotropy (fourth column) and average in-plane vorticity (last column). First row shows the corresponding map past variant $\mathrm{A}$, second row does past variant $\mathrm{B}$ and the last row shows comparison of both variants in term of isolines of the quantity above. $U_{r}$ is the reference velocity of empty wind-tunnel, in this case it is $11.5 \mathrm{~m} / \mathrm{s}$.

In order to catch some rough figure of the flow behavior past the airfoil, we present here the ensemble average quantities in Figure 2. Turbulent kinetic energy (third column in Figure 2, TKE) is calculated only on basis of the two measured velocity component:

$$
E_{T}=\frac{1}{2}\left(\sigma^{2}(u)+\sigma^{2}(v)\right)
$$

where

$$
\sigma(u)=\sqrt{\left\langle u^{2}\right\rangle-\langle u\rangle^{2}}
$$

\footnotetext{
${ }^{3}$ it is a standard description of the trajectory of the extruder of 3D printer, it is evolved from similar language used for CNC (Computer Numerical Control) milling machines.
} 
and $\sigma(v)$ analogically; $\langle\cdot\rangle$ denotes ensemble averaging. Note, that this value of TKE is underestimated by the entire contribution from third velocity component and by the the contribution of length-scales smaller than the single grid cell, which, in this case, has size $532 \times 532 \mu \mathrm{m}$.

The fourth column of Figure 2 shows the Degree of anisotropy defined in the book [30] and used e.g. in articles [31] or [32]. Each of the mentioned literature uses slightly different definition, we keep that of Gallego et al. [31], who measured turbulence statistics past a high-lift airfoil FX 63-137, only the nomenclature is different ( $u$ component means $v$ in Gallego's work.)

$$
\mathrm{DA}=\frac{\sigma^{2}(u)-\sigma^{2}(v)}{\sigma^{2}(u)}
$$

Its spatial distribution shows that the narrow central part of the wake is dominated by the fluctuations in span-wise direction (negative DA, red colors in Figure 2), while in the area around it, there is dominant the stream-wise component (positive DA, blue-like colors in figure). The dominance of stream-wise component is typical for continuing boundary layer, where the fluctuations on span-wise direction are damped by the presence of solid wall; this character of turbulence, when formed, continues into the wake of so-calledstreamed body. The dominance of span-wise fluctuations is typical for wake past so-called bluff body, where the von Kármán vortex street forms [33] due to the Kelvin-Helmholtz instability between the layer decelerated by the presence of body and the surrounding faster fluid.

The geometrical difference between products $\mathrm{A}$ and $\mathrm{B}$ imprints into the wake structure as follows: shorter trailing edge of A causes, that the edge is shifted to the suction side (up in our figures), because this edge is slanted toward the pressure side. The consequence is, that the entire wake structure past A is shifted towards the suction side by more than $1 \mathrm{~mm}$ (this is best apparent in the plot of average stream-wise velocity $u$, first column of Figure 2). The trailing edge of A has larger radius, therefore the area of separated flow is larger, which is visible at the thickness of the central strip of negative DA (fourth column of Figure 2). On the other hand, the variant B is little bit thicker (by the Minkowski sum with $0.1 \mathrm{~mm}$ circle), therefore the wake is larger, which is visible in the map of stream-wise velocity $u$ and turbulent kinetic energy.

The most interesting question is, what do the vortices in the wake?

\subsection{Statistical properties of individual vortices}

Our individual vortex searching algorithm [18] is based on direct fitting 2D instantaneous fluctuating velocity field by the vortex model function, which has four fitting parameters: position $(x$ and $y$ ), vortex core radius $R$ and vortex core circulation $\Gamma$. The fitting is based on minimizing energy, however, the highest energy content has the homogeneous sliding component, additionally, the wake contains the strong shear layers at repeating positions. We use the Reynolds decomposition to remove these two contributions:

$$
\vec{u}^{\prime}(\vec{x}, t)=\vec{u}(\vec{x}, t)-\langle\vec{u}(\vec{x})\rangle_{t}-\langle\vec{u}(t)\rangle_{x},
$$

By using individual vortex approach, we notice, that there are vortices of both orientations across the entire wake, with dominant one orientation at the edge of the wake, as it is shown in Figure 3, while this information cannot be visible from the spatial distribution of average of some scalar quantity (e.g. vorticity in Figure 2 last column.). We see again, that the wake past variant $\mathrm{A}$ is shifted in respect to $\mathrm{B}$. The position distribution of positive vortices has slightly sharper maximum than the distribution of negative vortices, which is repeatable for both airfoil variants.

The distribution of vortex core radii is shown in form of probability density function (PDF) as there is large span of values and the histogram bins are unequal and therefore we have to normalize the amount of events in each bin by its width. Additionally, we normalize the weight of each vortex by the energy, which has been saved from the original velocity field. Comparison of both normalizations (just by number or by this energy) is shown in Figure 4. We observe the scaling of vortex core radius distribution to be $\sim R^{1.6}$ for smaller vortices and $\sim R^{-2}$ for larger ones for both variants of the airfoil. At this moment, we do not have any theoretical justification for these scalings.

The right panel of Figure 4 shows the development of average vortex core radius with streamwise distance along the wake. It is not surprise, that the vortex radii slightly increase, which 

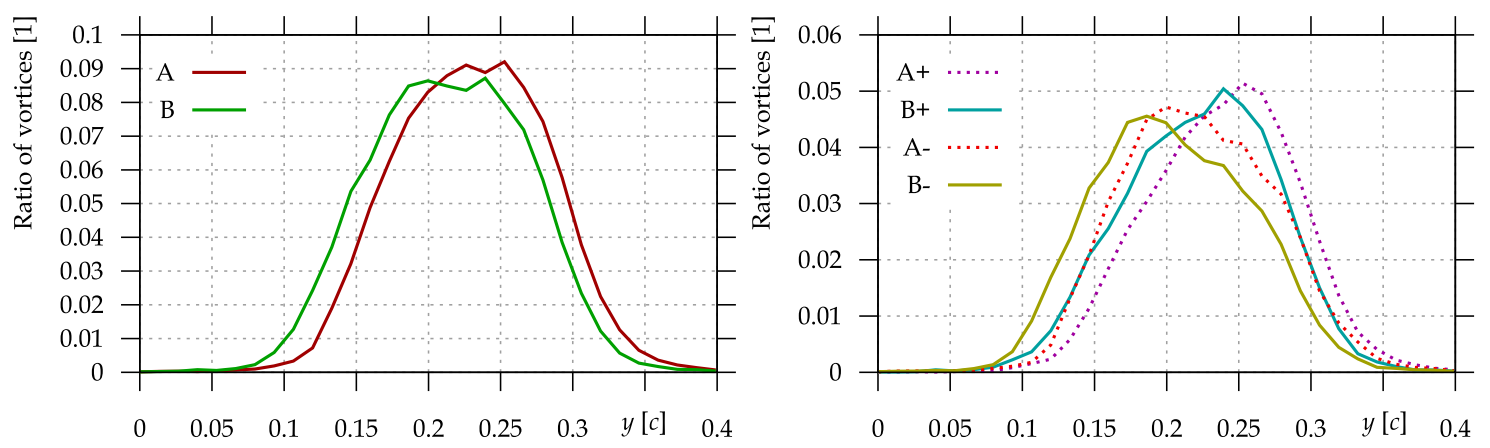

Figure 3: Histogram of vortex $y$-positions normalized by the number of all vortices. Left graph shows all vortices past variants $\mathrm{A}$ and $\mathrm{B}$, right graph shows positive and negative vortices separately. The $y$ coordinate is displayed in multiplies of $c$ counted from the edge of FoV.
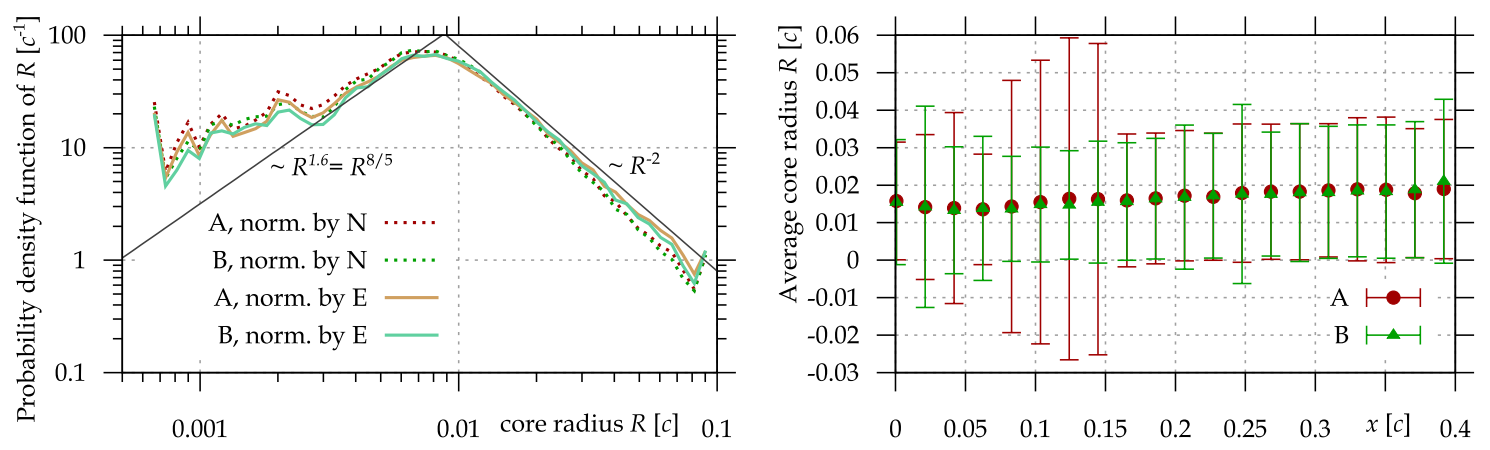

Figure 4: (Left) The distribution of the vortex core radii. The unequal bins are normalized by their width. The weight of vortices within the ensemble is 1 , denoted by doted lines. Or, they are normalized by the energy, they saved from the velocity field during fitting procedure, represented by solid lines. (Right) the ensemble average (normalized by 1 ) of vortex core radii as a function of the stream-wise distance from the beginning of FoV. All lengths are in $c$, the airfoil chord length.

corresponds to the general rules of turbulence decay. Here we note a strong difference between airfoil variants: the A variant displays s sudden increase in standard deviation of vortex core radii among vortices at around $0.15 c$ past the trailing edge. This can be explained by stronger tendency to formation of large-scale structure of von-Kármán vortex street in some snapshots.

Everyone tells, that larger vortices have larger energy. This is a logical assumption based on the fact, that larger vortex affects larger area, moreover, it is supported experimentally by the energy spectrum - larger fluctuations contains more energy. But it is not necessary that larger vortex can explain more energy in the decomposition of velocity field [18]. Figure 5 shows the dependence of energy saved during the fitting procedure from the velocity field as a function of vortex core radius. We see that (i) even small vortices can play a good game and (ii) the increase of efficiency has a certain maximum, above which the larger vortices are not able to fit into the velocity field. This can be caused by the finite size of velocity field (the vortex would have energy also outside of the FoV, but it does not count as we do not see it), but, in this case, it can be rather caused by the finite size of wake structure. On the other hand, the increase in vortex circulation ensures increase of useful energy (Figure 5 right).

The distance to nearest vortex is an ensemble characteristics and it displays a distribution with rather smooth increase to some maximal value followed by steep decrease signaling, that there is not many isolated vortices. Another interesting observation is, that the distance to nearest vortex of opposite orientation or the same orientation is almost equal ${ }^{4}$. It means, that there is

\footnotetext{
${ }^{4}$ correctly speaking, it is for A: $2.5 \pm 1.7 \%$ of $c$ the same orientation and $2.6 \pm 1.8 \%$ of $c$ for the opposite orientation,
} 

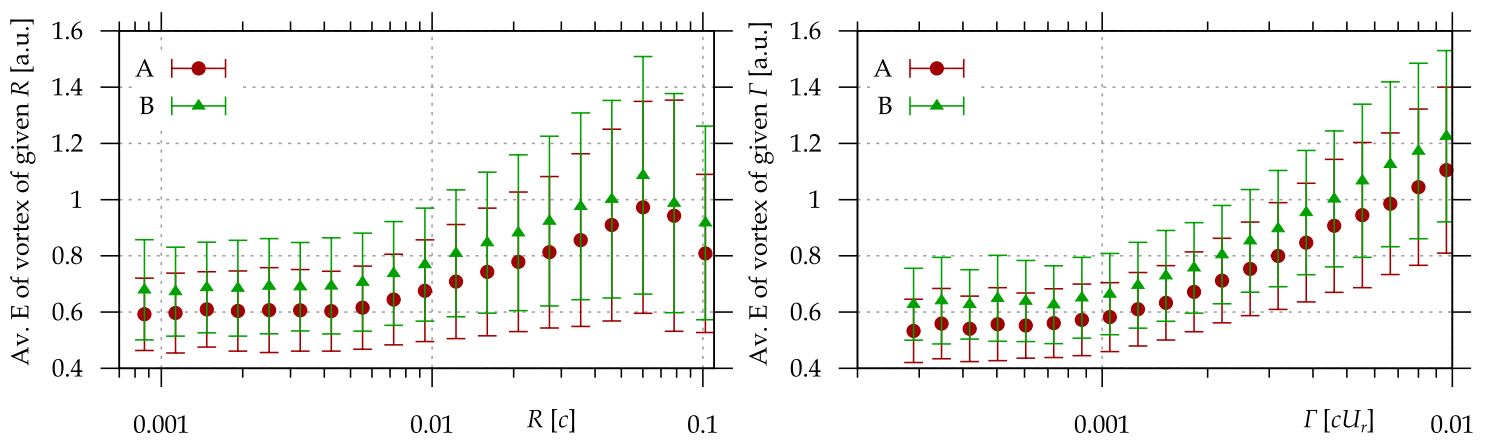

Figure 5: Average energy saved from the instantaneous fluctuating velocity field by vortices of different radii (left) or different circulations (right). Unit of radius is chord length $c$, circulation unit is $c \cdot U_{r}$, where reference velocity in empty wind tunnel is $U_{r}=11.5 \mathrm{~m} / \mathrm{s}$ in this case. Energy is plotted in arbitrary units without some link to the physical parameters of the experiment.
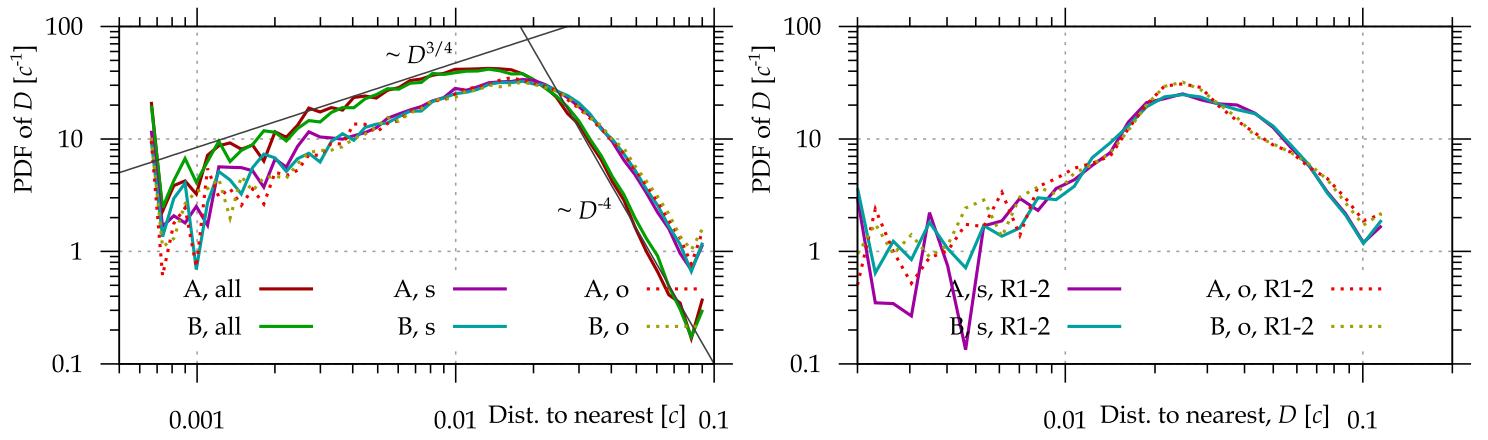

Figure 6: Distribution of the distance to the nearest other vortex, $D$. We can distinguish the vortices of same orientation (solid lines) or of the opposite orientation (doted lines) than the probed vortex. Left panel shows the entire ensemble, while the right one shows only distance between vortices within the same radius interval $1-2$ grid points, i.e. $6.7 \cdot 10^{-3}-1.3 \cdot 10^{-2} c$.

not some preferential grouping of vortices of the same orientation or some alternating grids in orientation. But the nearest vortex can be any vortex, small inside the core of large one, and so on. When we take into account only vortices of certain size interval - in Figure 6(right) there are only vortices with radius between $1-2$ grid points, i.e. $6.7 \cdot 10^{-3}-1.3 \cdot 10^{-2} c$. Then the situation is dramatically different: we see a significantly sharper peak in distribution of distance to nearest oppositely oriented vortex at distance around $0.025 \mathrm{c}$ than to a vortex of same orientation. This higher peak is followed by deeper depression in amount of distances between $0.03-0.06 c$.

\section{Conclusion}

We have developed an algorithm for direct vortex fitting of the experimentally obtained 2D velocity fields. This method is used for analyzing experimentally obtained instantaneous $2 \mathrm{D}$ velocity field past two slightly different realizations of a single airfoil. We explore the statistical distribution of vortex parameters: the vortex span-wise positions show the appearance of both vortex orientations across the wake with shifted maxima according to net vorticity spatial distribution. The vortex core radii display single maximum of occurrence at around $7 \cdot 10^{-3} c$, while the average is around $0.15 c$. This maximum is surrounded by polynomial dependencies of scalings $R^{1.6}$ and $R^{-2}$. The distribution of distance to nearest vortex displays scalings $D^{3 / 4}$ and $D^{-4}$. When we take into account only vortices of certain core radii, then there is observable asymmetry in the distributions

for B it is $2.6 \pm 1.7 \%$ of $c$ and $2.6 \pm 1.8 \%$ of $c$ 
of distance to nearest vortex of the same and opposite orientations suggesting some vague evidence of vortex ordering, which has to be explored in more details in the future.

\section{Acknowledgment}

The work was supported from ERDF under project Research Cooperation for Higher Efficiency and Reliability of Blade Machines (LoStr) No. CZ.02.1.01 / 0.0 / 0.0 / 16_026 / 0008389.

Author thanks to prof. Václav Uruba for valuable discussions, to Dr. Vitalii Yanovych for technical help, to Michaela Vacková for paying the conference fee, to doc. Petr Eret and Radka Lanči for supervising the above mentioned project.

\section{References}

[1] Barenghi, C. F. \& Skrbek, L., \& Sreenivasan, K. R.: Introduction to quantum turbulence. Proceedings of National Academy of Sciences of the United States of America, vol. 111 (2014) pp. 4647-4652.

[2] Fonda, E. \& Meichle, D. P. \& Ouellette, N. T. \& Hormoz, S., \& Lathrop, D. P.: Direct observation of Kelvin waves excited by quantized vortex reconnection. Proceedings of the National Academy of Sciences, vol. 111 no. Supplement_1: (2014) pp. 4707-4710.

[3] La Mantia, M. \& Skrbek, L.: Quantum, or classical turbulence?. EPL, vol. 105 no. 4: (2014).

[4] Alekseenko, S. V. \& Kuibin, P. A., \& Okulov, V. L.: Theory of concentrated vortices. Springer (2003).

[5] Amromin, E.: Analysis of vortex core in steady turbulent flow. Physics of Fluids, vol. 19 no. 11: (2007) pp. 13-16.

[6] Hnninen, R. \& Baggaley, A. W.: Vortex filament method as a tool for computational visualization of quantum turbulence. Proceedings of the National Academy of Sciences of the United States of America, vol. 111 no. SUPPL. 1: (2014) pp. 4667-4674.

[7] Varga, E. \& Babuin, S. \& Lvov, V. S. \& Pomyalov, A., \& Skrbek, L.: Transition to quantum turbulence and streamwise inhomogeneity of vortex tangle in thermal counterflow. Journal of Low Temperature Physics, vol. 187 no. 5-6: (2017) pp. 531-537.

[8] I.K., M. \& G.A., S., \& A., D. S.: On the algorithms for vortex element evolution modelling in 3d fully lagrangian vortex loops method. in Topical Problems of Fluid Mechanics 2020 pp. 152-159 (2020).

[9] Dergachev, S. A. \& Marchevsky, I. K., \& Shcheglov, G. A.: Flow simulation around 3d bodies by using lagrangian vortex loops method with boundary condition satisfaction with respect to tangential velocity components. Aerospace Science and Technology, vol. 94 (2019).

[10] K., K. \& I., M., \& I., S.: The high-accuracy numerical scheme for the boundary integral equation solution in 2 d lagrangian vortex method with semi-analytical vortex elements contribution accounting. in Topical Problems of Fluid Mechanics 2020 pp. 122-129 (2020).

[11] Holmén, V.: Methods for Vortex Identification. Lund University (2012). Master of Science Thesis.

[12] Ben-Gida, H. \& Liberzon, A., \& Gurka, R.: A stratified wake of a hydrofoil accelerating from rest. Experimental Thermal and Fluid Science, vol. 70 (2016) pp. 366-380.

[13] Kolář, V.: Vortex identification: New requirements and limitations. International Journal of Heat and Fluid Flow, vol. 28 no. 4: (2007) pp. 638-652.

[14] Maciel, Y. \& Robitaille, M., \& Rahgozar, S.: A method for characterizing cross-sections of vortices in turbulent flows. International Journal of Heat and Fluid Flow, vol. 37 (2012) pp. $177-188$. 
[15] Agrawal, A.: Measurement of spectrum with particle image velocimetry. Experiments in Flu$i d s$, vol. 39 no. 5: (2005) pp. 836-840.

[16] Agrawal, A. \& Prasad, A.: Properties of vortices in the self-similar turbulent jet. Experiments in Fluids, vol. 33 no. 4: (2002) pp. 565-577.

[17] Haller, G.: An objective definition of a vortex. Journal of Fluid Mechanics, vol. 525 (2005) pp. $1-26$.

[18] Duda, D.: Individual vortex searching algorithm. in Topical Problems of Fluid Mechanics 2020 pp. 56-63 (2020).

[19] Duda, D. \& Yanovych, V. \& Uruba, V. \& Němec, M., \& Žitek, P.: Vortices inside a singlestage axial air turbine captured by particle image velocimetry. MATEC Web of Conferences, vol. 328 (2020) p. 05002.

[20] Tropea, C. \& Yarin, A., \& Foss, J. F.: Springer Handbook of Experimental Fluid Mechanics. Springer, Heidelberg, DE (2007).

[21] Šulc, R. \& Ditl, P. \& Jašíkova, D. \& Kotek, M. \& Kopecký, V., \& Kysela, B.: Effect of particle image velocimetry setting parameters on local velocity measurements in an agitated vessel. Chemical Engineering and Technology, vol. 42 no. 4: (2019) pp. 827-834.

[22] Kellnerová, R. \& Fuka, V. \& Uruba, V. \& Jurčáková, K. \& Nosek, v. \& Chaloupecká, H., \& Jaňour, Z.: On street-canyon flow dynamics: Advanced validation of les by time-resolved piv. Atmosphere, vol. 9 no. 5: (2018).

[23] Uruba, V. \& Pavlík, D. \& Procházka, P. \& Skála, V., \& Kopecký, V.: On 3d flow-structures behind an inclined plate. in EPJ Web of Conferences vol. 143 (2017).

[24] Duda, D. \& Bém, J. \& Yanovych, V. \& Pavlíček, P., \& Uruba, V.: Secondary flow of second kind in a short channel observed by piv. European Journal of Mechanics, B/Fluids, vol. 79 (2020) pp. 444-453.

[25] Du, W. \& Zhao, Y. \& He, Y., \& Liu, Y.: Design, analysis and test of a model turbine blade for a wave basin test of floating wind turbines. Renewable Energy, vol. 97 (2016) pp. 414-421.

[26] "Airfoil Tools." http://airfoiltools.com. Accessed: 2020-05-30.

[27] Inkinen, S. \& Hakkarainen, M. \& Albertsson, A. ., \& Södergøard, A.: From lactic acid to poly(lactic acid) (pla): Characterization and analysis of pla and its precursors. Biomacromolecules, vol. 12 no. 3: (2011) pp. 523-532.

[28] Zhang, S.: Handbook of 3D Machine Vision: Optical Metrology and Imaging. CRC Press (2013).

[29] Krein, M. \& Šmulian, V.: On regularly convex sets in the space conjugate to a banach space. Annals of Mathematics, vol. 41 no. 3: (1940) pp. 556-583.

[30] Kundu, P. \& Cohen, I., \& Dowling, D.: Fluid Mechanics. Academic Press 6 ed. (2016).

[31] Sols-Gallego, I. \& Meana-Femndez, A. \& Femndez Oro, J. M. F. \& Argelles Daz, K. M. A., \& Velarde-Surez, S.: Turbulence structure around an asymmetric high- lift airfoil for different incidence angles. Journal of Applied Fluid Mechanics, vol. 10 no. 4: (2017) pp. 1013-1027.

[32] Porreca, L. \& Hollenstein, M. \& Kalfas, A. I., \& Abhari, R. S.: Turbulence measurements and analysis in a multistage axial turbine. Journal of Propulsion and Power, vol. 23 no. 1: (2007) pp. 227-234.

[33] Von Kármán, T.: Aerodynamics. McGraw-Hill paperbacks : science, mathematics and engineering McGraw-Hill (1963). 